\title{
Role of TCF7L2 and PPARG2 Gene Polymorphisms in Renal and Cardiovascular Complications among Patients with Type 2 Diabetes: A Cohort Study
}

\author{
Bancha Satirapoj Pamila Tasanavipas Ouppatham Supasyndh \\ Division of Nephrology, Department of Medicine, Phramongkutklao Hospital and College of Medicine, Bangkok, Thailand
}

\section{Keywords}

TCF7L2 gene · PPARG2 gene · Type 2 diabetes · Chronic kidney disease

\begin{abstract}
Background: The emerging renal and cardiovascular complications of type 2 diabetes (T2DM) genetics involves differently assembled gene variants including transcription factor 7-like 2 (TCF7L2) and peroxisome proliferator-activated receptor gamma 2 (PPARG2) polymorphisms. However, the relevance of these genes for complication prediction has not been extensively tested. Methods: We analyzed the SNP rs7903146 variants in TCF7L2 and PPARG2 gene polymorphisms for their contribution to the incidence of chronic kidney disease (CKD) and cardiovascular complications in a prospective cohort study. All T2DM patients were followed up to estimate the glomerular filtration rate and cardiovascular outcomes. Cox proportional hazards regression models were used to estimate the genotype effect on the incidence of CKD and vascular complications. Results: A total of 422 patients were included.
\end{abstract}

() 2019 S. Karger AG, Basel

E-Mail karger@karger.com www.karger.com/kdd
SNP rs7903146 variants in the TCF7L2 gene were classified into 3 groups: CC, 385 patients (91.2\%), CT, 32 patients (7.6\%), and TT, 5 patients (1.2\%), while in the PPARG2 gene they were classified into 2 groups: Pro12Pro, 404 patients (95.7\%) and Pro12Ala, 18 patients (4.3\%). The prevalence of CKD, cardiovascular disease, and death at the end of the 5 -year follow-up was $16.8,29$, and $7.9 \%$, respectively. The Pro12Ala variant of the PPARG2 gene was significantly associated with increased CKD risk at the end of the study (adjusted HR 3.45, 95\% Cl 1.01-11.77, $p=0.046$ ); it showed a significant association with increased cerebrovascular risk, but not cardiovascular disease and mortality. No genotype effect of rs7903146 in the TCF7L2 gene was apparent on renal and cardiovascular complications, except the TT variant of rs 7903146 increased cardiovascular events when compared with the non-TT variant. Conclusion: The findings of our study were that the Pro12Ala variant in the PPARG2 gene was associated with risk of developing CKD and cerebrovascular disease in Asian T2DM subjects in a prospective cohort study. The TCF7L2 polymorphism was not associated with cardiovascular outcomes.

(c) 2019 S. Karger AG, Basel
Bancha Satirapoj, MD

315, Division of Nephrology, Department of Medicine Phramongkutklao Hospital and College of Medicine

Rajavithi Road, Bangkok 10400 (Thailand)

E-Mail satirapoj@yahoo.com 


\section{Background}

Diabetes mellitus is a common disease present in about 415 million individuals worldwide and the number of subjects with diabetes aged $20-79$ years has been predicted to rise to 642 million by 2040 [1]. Patients with type 2 diabetes (T2DM) have increased cardiovascular disease, kidney disease, and patient mortality compared with the general population [2]. Pathogenetic factors of vascular complications regarding T2DM comprise hemodynamic factor, metabolic stimuli, and genetic susceptibility. T2DM with macrovascular and microvascular complications is a classic complex trait, whose development in a given individual likely reflects contributions from multiple genes whose expression is modulated by environmental factors $[3,4]$.

Genome-wide association studies have disclosed chromosomic regions containing diabetes mellitus susceptibility genes in chromosome $10 \mathrm{q}$, which were later defined as local variations in the transcription factor 7-like 2 (TCF7L2) gene, especially rs7903146 (T allele), which is associated with the strongest risk of developing T2DM [5]. In addition, the peroxisome proliferator-activated receptor gamma 2 (PPARG2) gene, located on chromosome $3 \mathrm{p}$, is associated with developing T2DM and vascular complications. A common $\mathrm{C}$ to $\mathrm{G}$ base exchange leads to the substitution of proline to alanine in codon 12 of exon 2 in the PPARG2 gene (the Pro12Ala polymorphism). This substitution is within the domain that enhances ligand-independent activation, and a functional study has shown that the Ala isoform leads to less efficient stimulation of PPARG2 target genes associated with increased risk of T2DM $[6,7]$.

Though the results of studies of TCF7L2 and PPARG2 polymorphisms and diabetic complications have been conflicting, several studies have suggested an association of the rs7903146 (T allele) of TCF7L2 and the Pro12Ala allele of the PPARG2 gene with vascular complications and mortality regarding T2DM $[8,9]$. In this study, we aimed to study the influence of the TCF7L2 rs7903146 and PPARG2 Pro12Ala polymorphisms on the risk of cardiovascular disease, renal complications, and mortality among Asian individuals with T2DM.

\section{Methods}

\section{Subjects}

The study employed a prospective cohort design. The patients were followed up from May 2011 to December 2015. Inclusion cri- teria of the study included age 18 years or older, and having T2DM. Exclusion criteria included active malignancy, severe heart, lung, or liver disease, previous stroke, chronic infection, e.g., tuberculosis within 1 year of starting the study, and any immunological or inflammatory disorders. All patients with T2DM aged $>18$ years attending the outpatient clinic at Phramongkutklao Hospital were invited to participate in the study of genetic risk factors for developing diabetic micro- and macrovascular complications. T2DM was diagnosed according to the 2015 American Diabetes Association diagnostic criteria for diabetes. A complete medical history was taken, a physical examination was conducted, and phenotypic characteristics were recorded for all subjects.

\section{Laboratory Testing}

All of the patients underwent a standardized clinical and laboratory evaluation. The serum concentration of creatinine was measured using the enzymatic method. Glomerular filtration rate (GFR) was estimated from calibrated serum creatinine using the 2009 Chronic Kidney Disease Epidemiology Collaboration (CKDEPI) creatinine equation, and hemoglobin A1c was determined by standard HPLC techniques.

\section{TCF7L2 and PPARG2 Polymorphism Genotyping}

Genomic DNA was extracted from $5 \mathrm{~mL}$ of peripheral blood using a standard salting-out procedure. Polymerase chain reaction (PCR) primers used for the s7903146 polymorphism of TCF7L2 were: rs7903146-F TTAGAGAGCTAAGCACTTTTTAGGTA and rs7903146-R ACTAAGTTACTTGCCTTCC. PCR primers used for Pro12Ala polymorphism of PPARG2 were: Pro12A-F GCCAATTCAAGCCCAGTC and Pro12A-R GATATGTTTGCAGACAGTGTATCAGTGAAGGAATCGCTTTCCG. The studied polymorphism was detected by PCR-restriction fragment length polymorphism assay (PCR-RFLP) (Taqman SNP genotype assay by ABI 7900HT Sequence Detection System). The quality control for these assays was assessed by randomly selecting 50 samples that were re-genotyped by an independent technician. Observed concordance between genotyping assays was $100 \%$.

\section{Clinical Outcomes}

Chronic kidney disease (CKD) was defined as having an estimated GFR $<60 \mathrm{~mL} / \mathrm{min} / 1.73 \mathrm{~m}^{2}$ and end-stage renal disease (ESRD) was defined as having an estimated GFR $<15 \mathrm{~mL} / \mathrm{min} /$ $1.73 \mathrm{~m}^{2}$ or requiring long-term renal replacement therapy. Cardiovascular events were defined as a new onset of acute coronary syndrome, myocardial infarction, unstable angina, or new cardiac procedure. Cerebrovascular events were defined as a new onset of transient ischemic attack, cerebral ischemia, or cerebral hemorrhage. The endpoints were all-cause mortality, cardiovascular events (including acute coronary syndrome, myocardial infarction, unstable angina, or new cardiac procedure) and renal events (including CKD and ESRD).

\section{Statistical Analysis}

Normally distributed variables are presented as mean $\pm \mathrm{SD}$, whereas nonnormally distributed variables are presented as median with interquartile range (IQR). Differences in categorical variables were tested for statistical significance using the $\chi^{2}$ test. For continuous variables the $t$ test and ANOVA were applied. All time-to-endpoint variables were analyzed using a log-rank test and displayed on Kaplan-Meier plots. Cox regression models were 

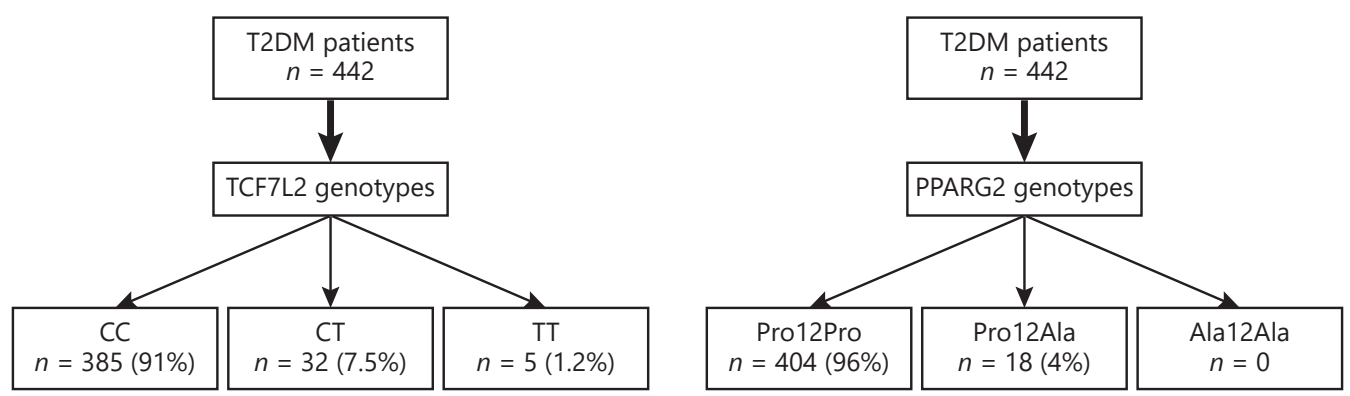

Fig. 1. Patients categorized by genotype.

used to estimate the unadjusted and adjusted hazard ratios (HR) with $95 \%$ confidence intervals (CI). A $p$ value of 0.05 or less was considered statistically significant. All calculations were performed using a commercially available program (SPSS for Windows, Version 15.0).

\section{Results}

\section{Baseline Characteristics}

The study enrolled 422 patients with T2DM according to PPARG2 and TCF7L2 genotypes. In the entire population, the Pro12Ala polymorphism of PPARG2 genotype frequencies were: Pro12Pro $=96 \%(n=404)$ and Pro12aAla $=4 \%(n=18)$. The rs7903146 polymorphism of TCF7L2 genotype frequencies were: CC $=$ $91 \%(n=385) ; \mathrm{CT}=8 \%(n=32)$, and TT $=1 \%(n=5)$ (Fig. 1).

This study population included $48.5 \%$ males with a mean age of $62 \pm 11.8$ years. Mean estimated GFR was $72.8 \pm 31.8 \mathrm{~mL} / \mathrm{min} / 1.73 \mathrm{~m}^{2}$. The prevalence of hypertension, dyslipidemia, and history of coronary artery disease was 95.0, 95.9, and 14.9\%, respectively. The baseline clinical and laboratory data of all patients according to genotype of PPARG2 and TCF7L2 polymorphisms are shown in Tables 1 and 2, respectively. No differences were found in genotype frequencies for age, sex, systolic blood pressure, diastolic blood pressure, LDL cholesterol, serum creatinine, and estimated GFR.

PPARG2 Polymorphism and Vascular Complications

During a median follow-up time of 60 (IQR 48-60) months, 67, 37, and 35 patients developed cardiovascular events, cerebrovascular events, and death, respectively. Variants of PPARG2 (Pro12Ala) were significantly associated with increased incidence of cerebrovascular events ( 22.2 vs. $8.1 \%, p=0.04)$, but not with the incidence of cardiovascular events and death (Table 3 ).

No difference in the incidence of ESRD was found by the PPARG2 genotype, but the incidence of ESRD (4.9\%) occurred only in the Pro12Pro genotype group. Further analysis was determined in a subgroup of patients with baseline GFR $>60 \mathrm{~mL} / \mathrm{min} / 1.73 \mathrm{~m}^{2}$ for exploring the incidence of $\mathrm{CKD}$. Among 375 patients with baseline estimated GFR $>60 \mathrm{~mL} / \mathrm{min} / 1.73 \mathrm{~m}^{2}$, patients with the Pro12Ala genotype developed CKD significantly more than those with the Pro12Pro genotype (75 vs. $49.3 \%, p=0.044)$. The Kaplan-Meier curve for the progression of CKD in the two genotype groups is presented in Figure 2. Patients with the Pro12Ala genotype were associated with a significantly higher incidence of CKD than patients with the Pro12Pro genotype ( $p=$ 0.034 , log-rank test). Finally, after adjusting for several established risk factors for developing kidney disease, only the Pro12Ala genotype (adjusted HR 3.75, 95\% CI 1.10-12.81, $p=0.047$ ), hypertension (adjusted HR $16.61,95 \%$ CI 1.98-123.29, $p=0.009$ ), and nonhypoglycemic agent (adjusted HR 3.45, 95\% CI 1.46-8.12, $p=$ $0.0095)$ were significantly and independently associated with CKD incidence (Table 4).

\section{TCF7L2 Polymorphism and Vascular Complications}

The TT variant of rs7903146 showed a significantly higher incidence of cardiovascular events (60 vs. $15.8 \%$, $p=0.01)$ compared with the non-TT variant of rs7903146. However, the TCF7L2 polymorphism was 
Table 1. Baseline characteristics categorized by PPARG2 genotype

\begin{tabular}{lccc}
\hline & $\begin{array}{c}\text { Pro12Ala } \\
(n=18)\end{array}$ & $\begin{array}{c}\text { Pro12Pro } \\
(n=404)\end{array}$ & $p$ value \\
\hline Male & $8(44.4)$ & $197(49.1)$ & 0.659 \\
Age, years & $62.9 \pm 8.8$ & $62.0 \pm 11.8$ & 0.742 \\
RAAS blockage & $9(50)$ & $281(69.9)$ & 0.074 \\
Aspirin & $18(100)$ & $327(81.3)$ & 0.053 \\
\hline Comorbid diseases & & & 0.609 \\
Hypertension & $17(94.4)$ & $384(95.1)$ & 1.000 \\
Dyslipidemia & $18(100)$ & $387(95.8)$ & 0.740 \\
Cardiovascular disease & $3(16.7)$ & $60(14.9)$ & 0.532 \\
\hline Clinical findings & & & 0.504 \\
Systolic blood pressure, $\mathrm{mm} \mathrm{Hg}$ & $133.9 \pm 4.9$ & $134.8 \pm 5.9$ & $82.2 \pm 5.4$ \\
Diastolic blood pressure, $\mathrm{mm} \mathrm{Hg}$ & $83.1 \pm 5.8$ & & 0.713 \\
\hline Laboratory findings & & & 0.304 \\
Fasting plasma glucose, $\mathrm{mg} / \mathrm{dL}$ & $132.3 \pm 32.3$ & $136.3 \pm 45.4$ & 0.456 \\
Hemoglobin Alc, \% & $6.9 \pm 1.2$ & $7.2 \pm 1.3$ & 0.064 \\
LDL, mg/dL & $97.7 \pm 21.0$ & $106.7 \pm 50.7$ & $73.3 \pm 25.2$ \\
\hline Estimated GFR, mL/min/1.73 m ${ }^{2}$ & $64.9 \pm 16.9$ & & \\
\hline
\end{tabular}

Data are presented as mean \pm SD or $n(\%)$, as appropriate. RAAS blockage, renin angiotensin aldosterone system blockage; LDL, low-density lipoprotein; GFR, glomerular filtration rate.

Table 2. Baseline characteristics categorized by TCF7L2 genotype

\begin{tabular}{lcccc}
\hline & $\begin{array}{l}\mathrm{CT} \\
(n=32)\end{array}$ & $\begin{array}{l}\mathrm{CC} \\
(n=385)\end{array}$ & $\begin{array}{c}\mathrm{TT} \\
(n=5)\end{array}$ & $p$ value \\
\hline Male & $17(53.1)$ & $187(48.9)$ & $1(20)$ & 0.312 \\
Age, years & $58.1 \pm 10.3$ & $62.3 \pm 11.8$ & $66.0 \pm 9.3$ & 0.110 \\
RAAS blockage & $20(62.5)$ & $267(69.7)$ & $3(60)$ & 0.634 \\
Aspirin & $24(75)$ & $317(82.3)$ & $4(80)$ & 0.540 \\
\hline Comorbid diseases & & & \\
Hypertension & $30(93.7)$ & $366(95.0)$ & $5(100)$ & 0.830 \\
Dyslipidemia & $28(87.5)$ & $372(96.6)$ & $5(100)$ & 0.037 \\
Cardiovascular disease & $3(9.38)$ & $57(14.8)$ & $3(60)$ & 0.012 \\
\hline Clinical findings & & & \\
Systolic blood pressure, $\mathrm{mm} \mathrm{Hg}$ & $133.5 \pm 5.9$ & $134.8 \pm 5.8$ & $139.8 \pm 1.5$ & 0.076 \\
Diastolic blood pressure, $\mathrm{mm} \mathrm{Hg}$ & $82.2 \pm 5.1$ & $82.3 \pm 5.4$ & $84.2 \pm 6.5$ & 0.726 \\
\hline Laboratory findings & & & \\
Fasting plasma glucose, $\mathrm{mg} / \mathrm{dL}$ & $140.2 \pm 38.9$ & $135.9 \pm 45.6$ & $123.4 \pm 24.8$ & 0.713 \\
Hemoglobin Alc, \% & $7.2 \pm 1.8$ & $7.2 \pm 1.3$ & $7.3 \pm 1.1$ & 0.986 \\
LDL, mg/dL & $106.7 \pm 37.4$ & $105.9 \pm 50.8$ & $131.4 \pm 38.1$ & 0.525 \\
Estimated GFR, mL/min/1.73 m ${ }^{2}$ & $72.3 \pm 31.8$ & $72.8 \pm 24.4$ & $87.0 \pm 20.0$ & 0.526 \\
\hline
\end{tabular}

Data are presented as mean \pm SD or $n(\%)$, as appropriate. RAAS blockage, renin angiotensin aldosterone system blockage; LDL, low-density lipoprotein; GFR, glomerular filtration rate. 
Table 3. Incidence of vascular complications and mortality categorized by PPARG2 genotype during the 5-year follow-up

\begin{tabular}{|c|c|c|c|c|c|}
\hline Cardiovascular events & $4(22.2)$ & $63(15.6)$ & 0.506 & 1.58 & $0.50-5.02$ \\
\hline Cerebrovascular events & $4(22.2)$ & $33(8.1)$ & 0.040 & 2.34 & $0.64-8.56$ \\
\hline Chronic kidney disease & $12(75)$ & $177(49.3)$ & 0.040 & 1.52 & $1.12-2.05$ \\
\hline$>10 \%$ of baseline & $13(72.2)$ & $230(56.9)$ & 0.209 & 2.05 & $0.66-6.41$ \\
\hline$>25 \%$ of baseline & $4(22.2)$ & $111(27.5)$ & 0.591 & 0.73 & $0.23-2.29$ \\
\hline Average GFR change per year, $\mathrm{mL} / \mathrm{min} / 1.73 \mathrm{~m}^{2}$ & $5.2 \pm 4.36$ & $5.18 \pm 7.34$ & 0.986 & 1.01 & $0.94-1.07$ \\
\hline End-stage renal disease & $0(0)$ & $20(4.9)$ & 1.000 & 0.01 & $0.16-8.04$ \\
\hline Mortality & $2(11.1)$ & $33(8.1)$ & 0.654 & 1.41 & $0.31-64.3$ \\
\hline
\end{tabular}

Data are presented as mean \pm SD or $n(\%)$, as appropriate. Percentage indicates the percentage of incidence within 5 -year comparison between genotype groups. Incidence was calculated by $\chi^{2}$ test, and Cox regression models were used to estimate the hazard ratios with $95 \%$ CI. Significant was defined as $p$ value $<0.05$.
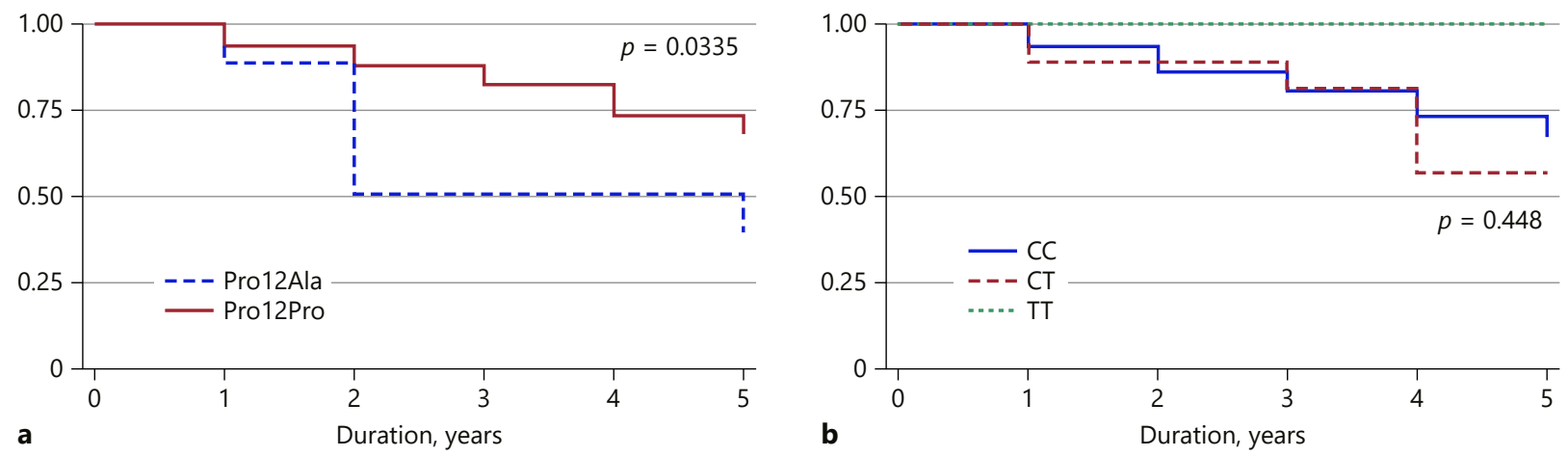

Fig. 2. Kaplan-Meier values of PPARG2 and TCF7L2 genotypes on renal survival among 268 patients with T2DM after 5 years of follow-up. a Pro12Ala had a significantly higher incidence of CKD $(p=0.0335)$. b No difference in incidence of CKD was found between TCF7L2 genotypes.

not associated with increased risk of cardiovascular events, cerebrovascular events, and death using Cox regression models (Table 5). However, no difference in the incidence of CKD was found between the TCF7L2 genotype (TT genotype: $0 \%$, CT genotype: $30 \%$, and CC genotype: $25.4 \%, p=0.452$ ) and ESRD (TT genotype: $0 \%$, CT genotype: $9.3 \%$, and CC genotype: $4.4 \%$, $p=1.000)$.

\section{Discussion}

This study, conducted in a representative sample of patients with T2DM, showed that the Pro12Ala polymorphism of PPARG2 was significantly associated with increased incidence of CKD and the Pro12Ala variant had a significantly higher incidence of cerebrovascular events. The Pro12 allele is more common in the Thai population 
Table 4. Cox proportional hazards modeling for developing CKD

\begin{tabular}{llll}
\hline Predictor variables & $\begin{array}{l}\text { Adjusted } \\
\mathrm{HR}^{1}\end{array}$ & $95 \% \mathrm{CI}$ & $p$ value \\
\hline Pro12Ala & 3.75 & $1.10-12.81$ & 0.035 \\
Hypertension & 16.61 & $1.98-123.29$ & 0.009 \\
Hyperlipidemia & 4.72 & $0.96-23.08$ & 0.056 \\
Hemoglobin A1c & 0.98 & $0.83-1.16$ & 0.813 \\
Nonhypoglycemic agent & 3.45 & $1.46-8.12$ & 0.005 \\
\hline
\end{tabular}

${ }^{1}$ Adjusted for hypertension, hyperlipidemia, hemoglobin A1c, and nonhypoglycemic agents.

Table 5. Incidence of vascular complications and mortality categorized by TCF7L2 genotype during the 5-year follow-up

\begin{tabular}{|c|c|c|c|c|c|c|c|}
\hline & $\begin{array}{l}\text { CC } \\
(n=385)\end{array}$ & $\begin{array}{l}\mathrm{CT} \\
(n=32)\end{array}$ & $\begin{array}{l}\mathrm{TT} \\
(n=5)\end{array}$ & $p$ value & $\begin{array}{l}\text { Hazard ratio } \\
\text { (CC vs. CT) }\end{array}$ & $95 \% \mathrm{CI}$ & $p$ value \\
\hline Cardiovascular events & $61(15.8)$ & $3(9.3)$ & $3(60)$ & 0.016 & 1.04 & $0.98-1.11$ & 0.329 \\
\hline Cerebrovascular events & $34(8.8)$ & $3(9.3)$ & - & 0.780 & 0.99 & $0.90-1.1$ & 0.917 \\
\hline Chronic kidney disease & $174(50.6)$ & $15(55.6)$ & - & 0.300 & 0.99 & $0.94-1.05$ & 0.854 \\
\hline End stage renal disease & $17(4.4)$ & $3(9.3)$ & - & 1.000 & 0.92 & $0.76-1.11$ & 0.207 \\
\hline Mortality & $33(8.6)$ & $2(6.2)$ & - & 0.716 & 1.02 & $0.94-1.12$ & 0.649 \\
\hline
\end{tabular}

Data are presented as $n$ (\%). Percentage indicates the percentage of incidence within 5-year comparison between genotype groups. Incidence was calculated by $\chi^{2}$ test, and Cox regression models were used to estimate the hazard ratios with $95 \%$ CI. Significant was defined as $p$ value $<0.05$.

than in Caucasian populations (98 vs. $88 \%$ ) [10]. In contrast, the TT genotype of TCF7L2 was associated with a significantly higher incidence of cardiovascular events during the 5 years of follow-up.

Individual susceptibility to diabetic nephropathy is well recognized even with the same environmental exposure. Polymorphisms of PPARG2 genes might be involved in the process of diabetic nephropathy. Expression of PPARG2 is found in the kidney including in the glomeruli, mesangial cells, and medullary collecting ducts [11, 12]. A related series of studies have investigated the association between the Pro12Ala polymorphism of PARG2 and diabetic nephropathy susceptibility, but provided inconclusive results [13-19]. Some studies reported that the Ala12 allele protected patients from worsening albuminuria and improving GFR in T2DM [14-16]. In contrast, in patients with type 1 diabetes with overt diabetic nephropathy, the Ala allele is associated with enhanced decline in GFR and predicts ESRD in Caucasian populations [17]. Similarly, studies in Asian populations found that the Pro12Ala polymorphism was significantly more likely to be associated with ESRD among patients without T2DM and predicted the mortality in patients with ESRD with T2DM [18]. Our study confirmed that the Pro12Ala variant in the PPARG2 gene was associated with increased incidence of CKD, but not associated with ESRD. Different ethnic compositions and genetic susceptibility to nephropathy of the populations studied may partly explain discrepancies in the findings.

PPARG2 has a key role in the molecular pathophysiology of lipid metabolism, obesity, and T2DM and is involved in regulating conventional cardiovascular risk factors [20]. More recent studies have indicated that PPARG2 regulates the transcription and expression of numerous target genes, which have been shown to be involved in adipocyte differentiation, oxidative stress, and atherosclerosis [21]. Two meta-analyses demonstrated that the PARG2 Pro12Ala polymorphism might be an inconsistent risk-conferring locus for the progression of coronary artery disease among Caucasians, but not among Asians 
$[22,23]$. It has been suggested the racial genetic diversity plays an important role in the etiology of atherosclerosis, similar to our findings in a Thai population. In addition, our study indicated that variants of PPARG2 (Pro12Ala) showed a significantly higher incidence of stroke than had been documented in related studies of patients with T2DM [24]. However, we found no association between the PPARG2 polymorphism and risk of stroke using Cox regression models. As to these findings, further studies are needed.

The TCF7L2 encoded protein TCF-4 plays a distinct role in the Wnt signaling pathway, which has been shown to take part in vascular remodeling, endothelial cell growth, and expression of several inflammatory genes implicated in atherosclerosis $[25,26]$. Several studies have reported the association of the TCF7L2 polymorphism rs7903146 with coronary artery disease and the progression of kidney disease in diabetic and nondiabetic subjects [27-31]. In contrast to related studies, in the ARIC study population, using more than 13,000 subjects, the TCF7L2 SNP rs7903146 did not demonstrate a significant association with incidence of coronary disease, ischemic stroke, peripheral artery disease, or all-cause mortality [32]. Our results did not show a significant association between TCF7L2 rs7903146 (TT genotype) with coronary heart disease and kidney disease in T2DM, but our study comprised a small number of patients with the TT genotype. The different results may be due to differences in race, daily activity, nutrient intake, and economic status. Considering that this constituted a genetic study of association, one limitation was the possibility of other nonevaluated variables that, by acting as confounders, could play significant roles in the findings.

The present study has several limitations. First, the number of patients with some variation of genotypes including TT and Pro12la polymorphism in the study were limited (TT group $n=5$, Pro12Ala group $n=18$ ). Second, limitations exist regarding the relative short period of follow-up and the small number of ESRD patients. The latter in general reduced the power to show a statistically significant relation. Third, data of albuminuria, hematuria, and imaging of kidneys are unavailable; the participants with GFR $\geq 60 \mathrm{~mL} / \mathrm{min} / 1.73 \mathrm{~m}^{2}$ and evidence of kidney damage might be classified in the non-CKD group in the analysis. Finally, we did not perform tests for silent cerebrovascular or cardiovascular disease in asymptomatic patients with T2DM. However, the findings have added extra evidence to the literature concerning this area for further research.

\section{Conclusion}

The findings of our study confirmed that the Pro12Ala variant in the PPARG2 gene was associated with the risk of developing CKD and cerebrovascular events in Asian T2DM subjects in the prospective cohort population. TCF7L2 polymorphism was not associated with cardiovascular outcomes. Further investigations are warranted to understand the pathway-based functional implications of the important loci in the PPARG2 gene.

\section{Acknowledgments}

We thank the medical staff at the Division of Nephrology, Phramongkutklao Hospital and College of Medicine. This study was supported by a grant from Phramongkutklao Hospital and College of Medicine and the National Science and Technology Development Agency (NSTDA), Bangkok, Thailand (grant No. P-13-00505).

\section{Statement of Ethics}

The study was performed in accordance with the Helsinki Declaration on Human Rights. The study was approved by the Institutional Review Board of the Royal Thai Army Medical Department, Bangkok, Thailand. All participants gave their written and informed consent.

\section{Disclosure Statement}

The authors declare that no potential conflict of interest exists.

References

Kidney Dis 2019;5:220-227 DOI: $10.1159 / 000497100$
1 Ogurtsova K, da Rocha Fernandes JD, Huang Y, Linnenkamp U, Guariguata L, Cho NH, et al. IDF Diabetes Atlas: global estimates for the prevalence of diabetes for 2015 and 2040. Diabetes Res Clin Pract. 2017 Jun;128:40-50.

2 Kornelius E, Chiou JY, Yang YS, Lu YL, Peng $\mathrm{CH}$, Huang CN: The Diabetes Shared Care Program and Risks of Cardiovascular Events in Type 2 Diabetes. Am J Med. 2015 Sep; 128(9):977-985. e3.

3 Satirapoj B, Adler SG. Comprehensive approach to diabetic nephropathy. Kidney Res Clin Pract. 2014 Sep;33(3):121-31.

4 Reddy MA, Zhang E, Natarajan R. Epigenetic mechanisms in diabetic complications and metabolic memory. Diabetologia. 2015 Mar; 58(3):443-55. 
5 Franklin CS, Aulchenko YS, Huffman JE, Vitart V, Hayward C, Polašek O, et al. The TCF7L2 diabetes risk variant is associated with $\mathrm{HbA}_{1}(\mathrm{C})$ levels: a genome-wide association meta-analysis. Ann Hum Genet. 2010 Nov; 74(6):471-8.

6 Costa V, Casamassimi A, Esposito K, Villani A, Capone M, Iannella R, et al. Characterization of a novel polymorphism in PPARG regulatory region associated with type 2 diabetes and diabetic retinopathy in Italy. J Biomed Biotechnol. 2009;2009:126917.

7 Liu L, Zheng T, Wang F, Wang N, Song Y, Li M, et al. Pro12Ala polymorphism in the PPARG gene contributes to the development of diabetic nephropathy in Chinese type 2 diabetic patients. Diabetes Care. 2010 Jan;33(1):144-9.

8 Bodhini D, Chidambaram M, Liju S, Prakash VG, Gayathri V, Shanthirani CS, et al. Association of TCF7L2 Polymorphism with Diabetic Nephropathy in the South Indian Population. Ann Hum Genet. 2015 Sep;79(5):373-9.

9 Ciccacci C, Di Fusco D, Cacciotti L, Morganti R, D’Amato C, Novelli G, et al. TCF7L2 gene polymorphisms and type 2 diabetes: association with diabetic retinopathy and cardiovascular autonomic neuropathy. Acta Diabetol. 2013 Oct;50(5):789-99.

10 Stumvoll M, Häring H. The peroxisome proliferator-activated receptor-gamma2 Pro12Ala polymorphism. Diabetes. 2002 Aug; 51(8):2341-7.

11 Asano T, Wakisaka M, Yoshinari M, Iino K, Sonoki K, Iwase M, et al. Peroxisome proliferator-activated receptor gammal (PPARgamma1) expresses in rat mesangial cells and PPARgamma agonists modulate its differentiation. Biochim Biophys Acta. 2000 Jun; 1497(1):148-54.

12 Yang T, Michele DE, Park J, Smart AM, Lin Z, Brosius FC 3rd, et al. Expression of peroxisomal proliferator-activated receptors and retinoid $\mathrm{X}$ receptors in the kidney. Am J Physiol. 1999 Dec;277(6):F966-73.

13 Yang B, Zhao H, Millward BA, Demaine AG. The Rate of Decline of Glomerular Filtration Rate May Not Be Associated with Polymorphism of the PPAR 22 Gene in Patients with Type 1 Diabetes and Nephropathy. PPAR Res. 2014;2014:523584.

$14 \mathrm{Yu}$ R, Bo H, Huang S. Association between the PPARG gene polymorphism and the risk of diabetic nephropathy: a meta-analysis. Genet Test Mol Biomarkers. 2012 May;16(5):429-34.
15 Caramori ML, Canani LH, Costa LA, Gross JL. The human peroxisome proliferator-activated receptor gamma2 (PPARgamma2) Pro12 Ala polymorphism is associated with decreased risk of diabetic nephropathy in patients with type 2 diabetes. Diabetes. 2003 Dec;52(12):3010-3.

16 Herrmann SM, Ringel J, Wang JG, Staessen JA, Brand E; Berlin Diabetes Mellitus (BeDiaM) Study. Peroxisome proliferator-activated receptor-gamma2 polymorphism Pro12Ala is associated with nephropathy in type 2 diabetes: The Berlin Diabetes Mellitus (BeDiaM) Study. Diabetes. 2002 Aug;51(8):2653-7.

17 Jorsal A, Tarnow L, Lajer M, Ek J, Hansen T, Pedersen $\mathrm{O}$, et al. The PPAR gamma 2 Pro12Ala variant predicts ESRD and mortality in patients with type 1 diabetes and diabetic nephropathy. Mol Genet Metab. 2008 Jul;94(3): 347-51.

18 Chao CT, Chen YC, Chiang CK, Huang JW, Hu FC, Fang CC, et al. Sequence variants of peroxisome proliferator-activated receptorgamma gene and the clinical courses of patients with end-stage renal disease. Dis Markers. 2015;2015:763459.

19 Lapice E, Monticelli A, Cocozza S, Pinelli $M$, Cocozza S, Bruzzese $D$, et al. The PPAR 22 Pro12Ala variant is protective against progression of nephropathy in people with type 2 diabetes. J Transl Med. 2015 Mar;13(1):85.

20 Guan Y. Peroxisome proliferator-activated receptor family and its relationship to renal complications of the metabolic syndrome. J Am Soc Nephrol. 2004 Nov;15(11):280115.

21 Spiegelman BM. PPAR-gamma: adipogenic regulator and thiazolidinedione receptor. $\mathrm{Di}$ abetes. 1998 Apr;47(4):507-14.

$22 \mathrm{Wu}$ Z, Lou Y, Jin W, Liu Y, Lu L, Lu G. The Pro12Ala polymorphism in the peroxisome proliferator-activated receptor gamma-2 gene $(\mathrm{PPAR} \gamma 2)$ is associated with increased risk of coronary artery disease: a meta-analysis. PLoS One. 2012;7(12):e53105.

23 Zafarmand $\mathrm{MH}$, van der Schouw YT, Grobbee DE, de Leeuw PW, Bots ML. Peroxisome proliferator-activated receptor gamma-2 P12A polymorphism and risk of acute myo- cardial infarction, coronary heart disease and ischemic stroke: a case-cohort study and meta-analyses. Vasc Health Risk Manag. 2008; $4(2): 427-36$.

24 Lee BC, Lee HJ, Chung JH. Peroxisome proliferator-activated receptor-gamma2 Pro12Ala polymorphism is associated with reduced risk for ischemic stroke with type 2 diabetes. Neurosci Lett. 2006 Dec;410(2):141-5.

25 Mulholland DJ, Dedhar S, Coetzee GA, Nelson CC. Interaction of nuclear receptors with the Wnt/beta-catenin/Tcf signaling axis: wnt you like to know? Endocr Rev. 2005 Dec; 26(7):898-915.

26 Wang X, Xiao Y, Mou Y, Zhao Y, Blankesteijn WM, Hall JL. A role for the beta-catenin/Tcell factor signaling cascade in vascular remodeling. Circ Res. 2002 Feb;90(3):340-7.

27 Muendlein A, Saely CH, Geller-Rhomberg S, Sonderegger G, Rein P, Winder T, et al. Single nucleotide polymorphisms of TCF7L2 are linked to diabetic coronary atherosclerosis. PLoS One. 2011 Mar;6(3):e17978.

28 Sousa AG, Marquezine GF, Lemos PA, Martinez E, Lopes N, Hueb WA, et al. TCF7L2 polymorphism rs7903146 is associated with coronary artery disease severity and mortality. PLoS One. 2009 Nov;4(11):e7697.

29 Buraczynska M, Swatowski A, MarkowskaGosik D, Kuczmaszewska A, Ksiazek A. Transcription factor 7-like 2 (TCF7L2) gene polymorphism and complication/comorbidity profile in type 2 diabetes patients. Diabetes Res Clin Pract. 2011 Sep;93(3):390-5.

30 Köttgen A, Hwang SJ, Rampersaud E, Coresh J, North KE, Pankow JS, et al. TCF7L2 variants associate with CKD progression and renal function in population-based cohorts. J Am Soc Nephrol. 2008 Oct;19(10):1989-99.

31 Luo Y, Wang H, Han X, Ren Q, Wang F, Zhang X, et al. Meta-analysis of the association between SNPs in TCF7L2 and type 2 diabetes in East Asian population. Diabetes Res Clin Pract. 2009 Aug;85(2):139-46.

32 Bielinski SJ, Pankow JS, Folsom AR, North KE, Boerwinkle E. TCF7L2 single nucleotide polymorphisms, cardiovascular disease and all-cause mortality: the Atherosclerosis Risk in Communities (ARIC) study. Diabetologia. 2008 Jun;51(6):968-70.
TCF7L2 and PPARG2 Gene

Polymorphisms in Type 2 Diabetes
Kidney Dis 2019;5:220-227

DOI: $10.1159 / 000497100$ 